\title{
Drug design, discovery and development and their safety or efficacy on human body
}

\author{
Shamima Shultana, Kazi M Maraz, Arwah Ahmed, Tanzila Sultana and Ruhul A Khan * \\ Institute of Radiation and Polymer Technology, Atomic Energy Research Establishment, Savar, Dhaka, Bangladesh.
}

GSC Advanced Research and Reviews, 2021, 17(02), 113-122

Publication history: Received on 07 October 2021; revised on 16 November 2021; accepted on 18 November 2021

Article DOI: https://doi.org/10.30574/gscbps.2021.17.2.0330

\begin{abstract}
Drug Design, often mentioned as rational drug design or just rational design. It is defined as the study of the shape of molecules in order to determine how they will bind receptors on cells or combine with other molecules. It is based on molecular shape or architecture is an alternative to blindly testing hundreds of molecules to see if one or more of them will bind cellular or molecular targets. The drug is an organic molecule, when it is bind to target site it can either inhibit or activate the function of a bio-molecule which results in therapeutic benefit.
\end{abstract}

Keywords: Definition; Molecular target; Discovery; New drugs investigation; Clinical research; Clinical trials

\section{Introduction}

Developing a new drug from an original idea to the launch of a finished product is a complex process which can take 12-15 years and cost in excess of \$1 billion. The idea for a target can come from a variety of sources including academic and clinical research and from the commercial sector. It may take many years to build up a body of supporting evidence before selecting a target for a costly drug discovery program. Once a target has been chosen, the pharmaceutical industry and, more recently, some academic centers would have streamlined a number of early processes to identify molecules which possess suitable characteristics to make acceptable drugs [1]. Drug development and discovery includes preclinical research on cell-based and animal models and clinical trials on humans, and finally move forward to the step of obtaining regulatory approval in order to market the drug. Modern drug discovery involves the identification of screening hits, medicinal chemistry and optimization of those hits to increase the affinity, selectivity (to reduce the potential of side effects), efficacy/potency, metabolic stability (to increase the half-life), and oral bioavailability. Once a compound that fulfills all of these requirements has been identified, it will begin the process of drug development prior to clinical trials [2].

This review will look at key preclinical stages of the drug discovery process, beginning with initial target identification and validation. The primary focus of this review is on general approaches and considerations toward development of analytical methods for separation, identification, and quantification of active pharmaceutical compounds (APIs), which may be applied within various functions in the drug development continuum. The review also discusses the issues and parameters that must be considered in the validation of analytical methods, clinical and pre-clinical study which helps to determine safety and efficacy of drug molecule on human body.

- The Discovery and Development

- Preclinical Research. Preclinical Research. Drugs undergo laboratory and animal testing to answer basic questions about safety.

- Clinical Research.

- FDA Review.

* Corresponding author: Ruhul A Khan
Institute of Radiation and Polymer Technology, Atomic Energy Research Establishment, Savar, Dhaka, Bangladesh.

Copyright $@ 2021$ Author(s) retain the copyright of this article. This article is published under the terms of the Creative Commons Attribution Liscense 4.0 
- $\quad$ FDA Post-Market. Safety Monitoring.

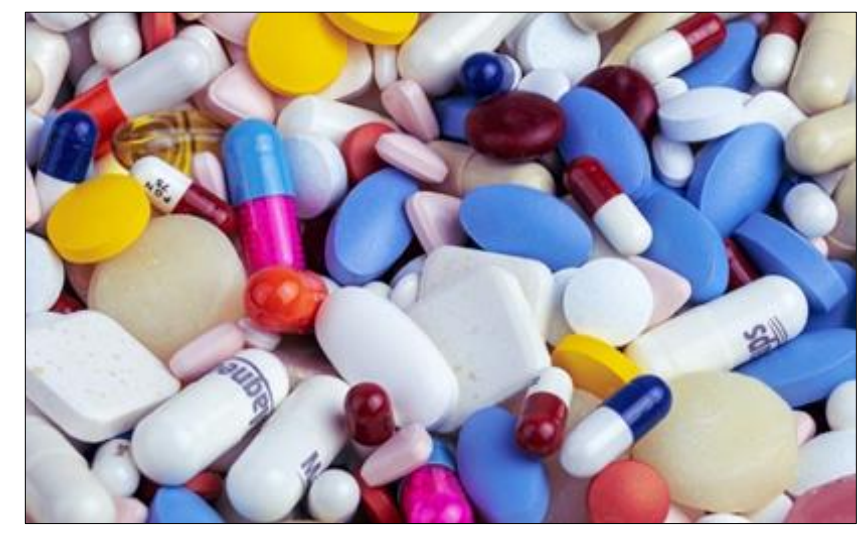

Figure 1 Drugs

\section{Drug Development Process}

\subsection{Drug discovery and development}

Discovery and development of new drugs is a very lengthy and costly process. In the research-based drug industry, $\mathrm{R}$ and $\mathrm{D}$ decisions have very long-term ramifications, and the impact of market or public policy changes may not be fully realized for many years. From both a policy perspective, as well as an industrial perspective, it is therefore important to continue to analyze the components of and trends in the costs of pharmaceutical innovation [3]. Drug development, from initial discovery of a promising target to the final medication, is an expensive, lengthy and incremental process. The ultimate goal is to identify a molecule with the desired effect in the human body and to establish its quality, safety and efficacy for treating patients. The latter requirements ensure that the approved medication improves patient's quality of life, not only by curing their illness, but also by making sure that the cure does not become the cause of other problems, namely side effects. It also means that this is a particularly costly and prolonged process. At present, bringing a single new drug to market costs around US\$800 million, an amount that doubles in every five years. According to the US Food and Drug Administration (FDA), it takes, on average, 12 years for an experimental drug to progress from bench to market [4].

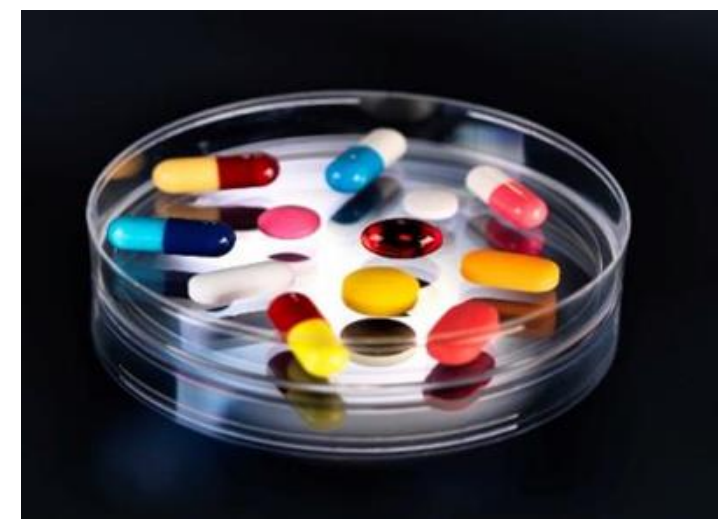

Figure 2 Drug appearance- Tablet and Capsule

The most and difficult part of the whole drug discovery procedure is the optimization of Absorption, Distribution, Metabolism, Excretion, and Toxicity (ADMET) properties of a drug. The ADMET profile has a major impact on producing a successful drug. The ADMET profile is discussed following:

\subsubsection{Absorption}

Absorption is the process of transferring a drug from its site of administration to the bloodstream. 


\subsubsection{Distribution}

When a drug is absorbed into the bloodstream, it can be carried throughout the body. This process is defined as a distribution and reversible process.

\subsubsection{Metabolism}

The drug is not readily metabolized. Drugs are removed from the body either unchanged through the kidneys and bile, or they may undergo chemical changes that allow them to be more easily excreted.

\subsubsection{Excretion}

The drug is not readily secreted. Basically drug excretion is a mechanism of eliminating a drug from the body. The greatest proportion of drugs are excreted through kidneys but there are some others route for eliminating such as lungs, milk, sweat, tears, skin, or saliva

\subsection{Toxicity}

The drugs may not affect all others tissue or cell. Drugs toxicity is also called Adverse Drug reaction (ADR).Drugs become toxins when a patient has accumulated overdose of drugs or drugs combine with other drugs that leads to the adverse effect like breathing suppression, lowers oxygen levels and finally fatal [5].

The following three factors affect the cost of drug development.

- Number of compounds synthesized: Of the about 5000-10,000 compounds studied, only one drug reaches the market.

- Nature of the lead molecule: Cost of production will be high if the lead molecule is prepared by an expensive route.

- Standards required for new drugs: The standards required by regulatory authorities prior to release of a drug into the market have increased dramatically. In the discovery phase, each drug cost about $\$ 350$ million. The Food and Drug Association processes I, II and III cost another $\$ 150$ million. This brings the total to about $\$ 500$ million for each drug put on to the market for consumers.

It is important to know the following ideal features of a drug molecule.

- $\quad$ Drug must be safe and effective

- Drug should have good bioavailability

- Drug must be metabolically stable and with a long half-life

- Drug should be nontoxic with minimal or no side effects

- Drug should have selective distribution to target tissues or disease state [6]

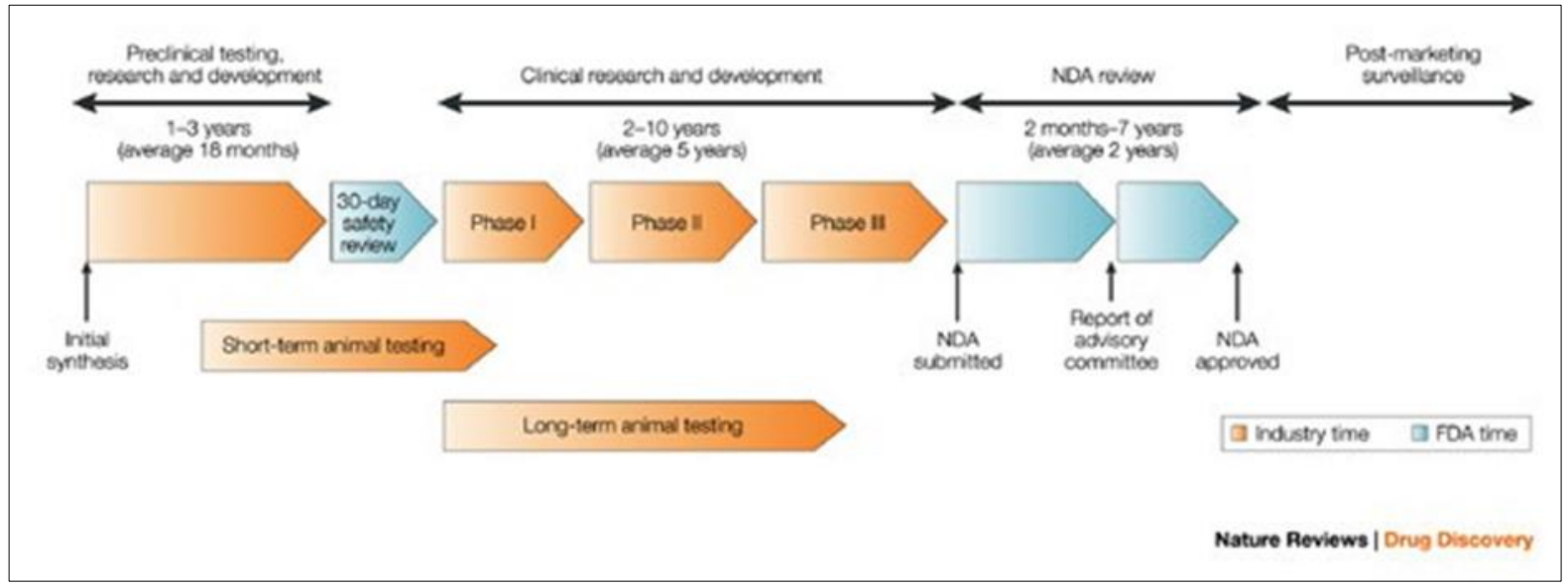

Figure 3 Drug approval process in the US [7]

Once a molecule is approved, it must be manufactured according to high standard of purity and stability as prescribed by regulations. Although manufacturing is not usually a concern for discovery biologists, the process of manufacturing a new medicine can be complex and expensive, particularly for biological products [8]. 
Stages of drug discovery and development include

- Target identification

- Target validation

- lead identification

- lead optimization

- Product characterization and Formulation and development

- Preclinical research

- Investigational New Drug

- New Drug Application

- Clinical trials

- Approval

Modern Technologies of Interest for the Process of Drug Development

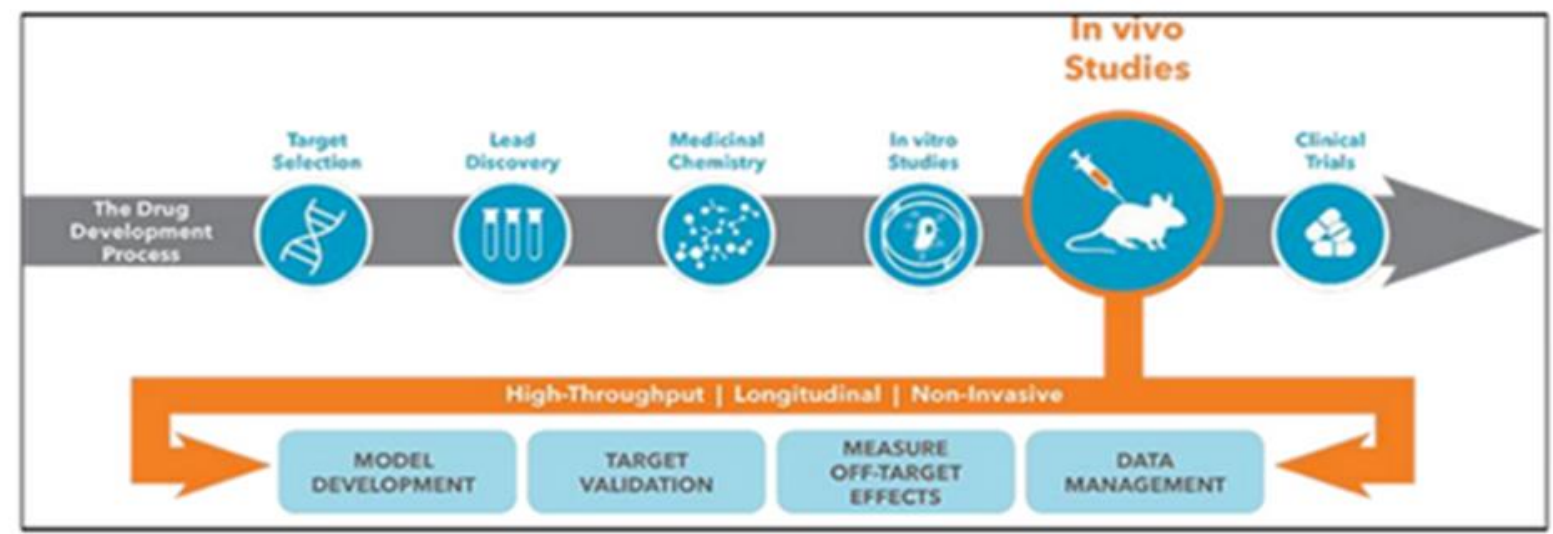

Figure 4 Stages of drug discovery and development process [9]

\subsection{Target identification}

Drugs fail in the clinic for two main reasons; the first is that they do not work and the second is that they are not safe. As such, one of the most important steps in developing a new drug is target identification and validation. A target is a broad term which can be applied to a range of biological entities which may include for example proteins, genes and RNA. A good target needs to be efficacious, safe, meet clinical and commercial needs and, above all, be 'druggable'. A 'drug-gable' target is accessible to the putative drug molecule, be that a small molecule or larger biological and upon binding, elicit a biological response which may be measured both in vitro and in vivo. It is now known that certain target classes are more amenable to small molecule drug discovery, for example, G-protein-coupled receptors (GPCRs), whereas anti- bodies are good at blocking protein/protein interactions. Good target identification and validation enables increased confidence in the relationship between target and disease and allows us to explore whether target modulation will lead to mechanism-based side effects [10].

\subsection{Target validation}

The drug target has to be validated experimentally according to the proposed mode of action. Here data link directly to the probability of clinical efficacy (i.e. experiments in human cells/tissues of eminent importance). Functional studies can apply genetic knockdown, knockout or, using target specific tools, if SMOL compounds or tool antibodies are available. In vitro cell-based mechanistic studies can be used to reveal regulative characteristics of targets and the pathways in which they are involved. Finally, depending on the disease, it can be necessary to evaluate the relevance of a particular target for the disease in appropriate animal models. Assuming that functional orthology between mice and humans is given, and suitable disease models exist, knockout or transgenic animals can be used for target validation. In general, however, the translation of in vivo validation into humans is not risk-free. Whereas some models promise to be highly predictive for the situation in humans, others show strong discrepancies.

Moreover, some diseases are restricted to higher primates, whereas most mechanistic animal studies are carried out in rodents. Hence, not all indication-specific challenges can be addressed at this early stage of drug discovery [11]. 
Target validation can be broken down in to two key steps.

Reproducibility: Once a drug target is identified, whether it be via a specific technique or from review of literature, the first step is to repeat the experiment to confirm that it can be successfully reproduced. The target validation technique includes affinity chromatography, expression-cloning, protein microarray, reverse transfected cell microarray, biochemical suppression, siRNA, DNA microarray, system biology and study of existing drugs [12,13].

Introduce variation to the ligand (drug)-target- environment

- Genetic manipulation of target genes (in vitro) knocking down the gene (shRNA, siRNA, miRNA), knocking out the gene (CRISPR), knocking in the genes (viral transfection of mutant genes)

- Antibodies interacting to the target with high affinity and blocking further interactions

- Chemical genomics chemical approaches against genome encoding protein [14].

\section{Identification of Lead}

A chemical lead is defined as a synthetically stable, feasible, and drug like molecule active in primary and secondary assays with acceptable specificity, affinity and selectivity for the target receptor. This requires definition of the structure activity relationship as well as determination of synthetic feasibility and preliminary evidence of in vivo efficacy and target engagement. Characteristics of a chemical lead are:

SAR defined

- $\quad$ Drug ability (preliminary toxicity, hERG)

- $\quad$ Synthetic feasibility

- Select mechanistic assays

- In vitro assessment of drug resistance and efflux potential

- Evidence of in vivo efficacy of chemical class

- $\quad$ PK/Toxicity of chemical class known based on preliminary toxicity or in silico studies.

In order to decrease the number of compounds that fail in the drug development process, a drug ability assessment is often conducted. This assessment is important in transforming a compound from a lead molecule into a drug. For a compound to be considered druggable it should have the potential to bind to a specific target; however, also important is the compound's pharmacokinetic profile regarding absorption, distribution, metabolism, and excretion. Other assays will evaluate the potential toxicity of the compound in screens such as the Ames test and cytotoxicity assay [15].

\subsection{Lead candidate optimization}

Molecules, identified as "Leads" in the previous stage, are subjected to optimization work. This step is believed to be essential in contributing towards drug discovery process. At this stage, leads are modified to provide "best" analogues displaying improved potency, efficacy, pharmacokinetic and pharmacodynamic properties. The changes are accomplished by chemical modifications chosen by structure activity analysis. If a target structure is known, structurebased design could also be employed in introducing the changes. As this process involves simultaneous optimization of multiple parameters, it is quite time consuming and a costly step. In the entire drug discovery process lead optimization step is thought to be a rate-limiting step [16].

\subsection{Product characterization and Formulation and development}

Usually molecule is characterized by its size, shape, strength, weakness, use, toxicity, and biological activity after showing a promising therapeutic activity. The drug product is categorized according to its route of administration. A description of the drug product qualitative/quantitative composition provides a list of all ingredients, including solvents used in the manufacture of the drug product. The functional aspects of each component of the drug product are central to the development rationalization of the formulation and serve as reference points in the examination of supportive development data.

Formulations can be categorized according to the route of administration and include oral, rectal, vaginal, inhalation, topical, transdermal, intraocular, intranasal, and parenteral drug products. Pharmaceutical development information provides the scientific rationale for the formulation development approach through to the final development and justification of a suitable dosage form. Regulatory guidance describes only limited detail of the requirements for the data sets associated with pharmaceutical development. Although more detailed guidance is available for the toxicological assessment of excipients, the detailed scientific approach to formulation development and justification is 
left to the discretion of the development organization, and the level of detail is dependent upon the complexity of the dosage form [17].

Once researchers identify a promising compound for development, they conduct experiments to gather information on:

- How it is absorbed, distributed, metabolized, and excreted

- Its potential benefits and mechanisms of action

- The best dosage and best way of administration

- $\quad$ Side effects (often referred to as toxicity)

- How it affects different groups of people (such as by gender, race, or ethnicity) differently

- How it interacts with other drugs and treatments

- Its effectiveness as compared with similar drug [18].

\section{Preclinical research}

Preclinical studies are in vivo studies that usually occur in animals. Initially, they are conducted in animals without the disease of interest in order to test toxicology, then later in animal models of the disease that allow for assessment of disease-modifying effects. The purpose of this research is to obtain FDA approval for an IND that will in turn allow for subsequent research in human subjects.

Testing must typically occur in two different mammalian species, as per FDA guidelines. The tests are often conducted by a center with expertise in this area; academic centers with veterinary schools may offer this service, as well as specialized outside companies. Note that the core preclinical studies must be completed before the compound is given to any human subjects. These studies can help determine the no-observed-adverse-effect levels (NOAELs) for the compound, which are necessary to identify the initial dose level of the compound for subsequent Phase 1 human clinical trials (first-in-man). Failure to follow FDA guidelines for conducting preclinical evaluations may result in an IND application being rejected, and culminate in the need to repeat the testing, potentially leading to significant delays in drug development. These FDA guidelines include rigorous quality control, oversight, and comprehensive reporting of results. This can be particularly challenging for academic entrepreneurs because, in some cases, this initial work would have been done in an exploratory capacity and thus may not meet FDA guidelines. On average, the preclinical phase can take three and a half years.

It is also worth noting that these preclinical studies may suggest a potential role for the compound in animal diseases and thus a veterinary medicine application for the compound. This offers several important benefits, including the potential to gather additional safety data and faster access to the veterinary market as compared to human applications, which can lead to revenue from sales in the Veterinary medicine market that may support further development in humans [19].

\subsection{Investigational New Drug (IND) Application}

It's an application filed to the FDA to start clinical trials in humans if the drug was found to be safe from the reports of Preclinical trials. The IND application should provide high excellence pre-clinical data to justify the testing of the drug in humans. Almost $85 \%$ of drugs are subjected to clinical trials, for which IND applications are filed. An institution, called a Sponsor, is responsible for submitting the IND application

A Pre - IND assessment can be organized with the FDA to deliberate multiple issues:

- The design of animal research, which is required to lend support to the clinical studies

- The intended protocol for conducting the clinical trial

- The chemistry, manufacturing, and control of the investigational drug [20].

\section{New Drug Application}

If a drug appears promising in pre-clinical studies, a drug sponsor or sponsor-investigator can submit an investigational new drug (IND) application. This detailed proposal contains investigator qualifications and all pre-clinical drug information and data, and a request for exemption from the federal statutes that prohibit interstate transport of unapproved drugs. After approval, the drug is studied and if demonstrated safe and efficacious in the intended population, the drug sponsor can then submit a New Drug Application (NDA) to the FDA. After an extensive review by the FDA that often involves a recommendation by an external committee, the FDA determines whether the therapeutic 
can be granted an indication and marketed. After final approval, the drug can continue to be studied in phase IV trials, in which safety and effectiveness for the indicated population is monitored. To facilitate evaluation and endorsement of foreign drug data, efforts have been made to harmonize this approval process across the United States, Europe, and Japan through the International Conference on Harmonization of Technical Requirements for Registration of Pharmaceuticals for Human Use (ICH) [21].

\subsection{Clinical trials}

Clinical trials may be defined as the process designed to determine the safety and efficacy of a particular drug or device on humans. According to WHO, the clinical trial is any research study that prospectively assigns human to one or more health-related interventions to evaluate the effects on health outcomes. Generally, clinical trials have been performed when the satisfactory information regarding the quality of non-clinical safety are available which have been approved by governed authority of drug or device. Initially, the trials have been known to depend upon the product quality conjunction with the various stages involved in the development of product. Initially, the investigators select the volunteers or patients into small quantities and conduct the clinical trials. Once, the positive data have been collected regarding safety and efficacy, the patient number is increased. Moreover, the clinical trials have been performed in several countries. In addition, clinical trials include new drugs that can be classified into four phases. Each of phases is treated as a separate clinical trial for the approval of drugs.

\subsection{The table of phases effectivity}

Table 1 Phases of Clinical Trials [22]

\begin{tabular}{|c|c|c|c|c|c|}
\hline Phases & Primary goal & Dose & $\begin{array}{l}\text { Monitoring of } \\
\text { patients }\end{array}$ & $\begin{array}{l}\text { Number of } \\
\text { Participants }\end{array}$ & Notes \\
\hline Preclinical & $\begin{array}{l}\text { Non-human efficacy, } \\
\text { toxicity and } \\
\text { pharmacokinetic and } \\
\text { information }\end{array}$ & Unrestricted & Researcher & $\begin{array}{l}\text { Invitro, invivo } \\
\text { animal }\end{array}$ & \\
\hline Phase 0 & $\begin{array}{l}\text { Pharmacokinetic and } \\
\text { Pharmacodynamic }\end{array}$ & $\begin{array}{l}\text { Very small sub } \\
\text { therapeutic }\end{array}$ & $\begin{array}{l}\text { Clinical } \\
\text { researcher }\end{array}$ & 10 people & $\begin{array}{l}\text { Often skip from } \\
\text { this phase }\end{array}$ \\
\hline Phase I & $\begin{array}{l}\text { Testing of drugs on } \\
\text { healthy volunteers for } \\
\text { dose ranging }\end{array}$ & $\begin{array}{l}\text { Often sub } \\
\text { therapeutic but } \\
\text { with ascending } \\
\text { dose }\end{array}$ & $\begin{array}{l}\text { Clinical } \\
\text { researcher }\end{array}$ & 20-100 people & $\begin{array}{l}\text { Determines } \\
\text { that whether } \\
\text { the drug is safe } \\
\text { and efficient }\end{array}$ \\
\hline Phase II & $\begin{array}{l}\text { Testing of drugs on } \\
\text { patients to asses } \\
\text { efficacy and safety }\end{array}$ & $\begin{array}{l}\text { Therapeutic } \\
\text { dose }\end{array}$ & $\begin{array}{l}\text { Clinical } \\
\text { researcher }\end{array}$ & $100-300$ people & $\begin{array}{l}\text { Determines } \\
\text { whether the } \\
\text { drug can have } \\
\text { an efficacy }\end{array}$ \\
\hline Phase III & $\begin{array}{l}\text { Testing of drugs on } \\
\text { patient to asses efficacy } \\
\text { and safety }\end{array}$ & $\begin{array}{l}\text { Therapeutic } \\
\text { dose }\end{array}$ & $\begin{array}{l}\text { Clinical } \\
\text { researcher and } \\
\text { personal } \\
\text { physician }\end{array}$ & $\begin{array}{l}1000-2000 \\
\text { people }\end{array}$ & $\begin{array}{l}\text { Determines } \\
\text { therapeutic } \\
\text { effect of drug }\end{array}$ \\
\hline Phase IV & $\begin{array}{l}\text { Post marketing } \\
\text { survillence- } \quad \text { watching } \\
\text { drug use in public }\end{array}$ & $\begin{array}{l}\text { Therapeutic } \\
\text { dose }\end{array}$ & $\begin{array}{l}\text { Personal } \\
\text { physician }\end{array}$ & $\begin{array}{l}\text { Anyone seeking } \\
\text { treatment form } \\
\text { their physician }\end{array}$ & $\begin{array}{ll}\text { Watch } & \text { drug } \\
\text { long } & \text { term } \\
\text { effects } & \end{array}$ \\
\hline Phase V & Translational research & No dosing & None & All report used & $\begin{array}{l}\text { Research on } \\
\text { date collected }\end{array}$ \\
\hline
\end{tabular}

\subsubsection{Phase 0}

Phase 0 is a recent designation for exploratory, first-in-human trials conducted in accordance with the U.S. Food and Drug Administration's (FDA) 2006 Guidance on Exploratory. Investigational New Drug (IND) Studies Phase 0 trials are designed to speed up the development of promising drugs or imaging agents by establishing very early on whether the drug or agent behaves in human subjects as was anticipated from preclinical studies. Distinctive features of Phase 0 trials include the administration of single sub therapeutic doses of the study drug to a small number of subjects (10 to 
15) to gather preliminary data on the agent's pharmacokinetics (how the body processes the drug) and pharmacodynamics (how the drug works in the body) [23].

Phase 0 studies do not provide any specific data about the safety and efficacy of the test drug. Furthermore, the drug development companies have been noted to perform Phase 0 studies for ranking the drug candidate in order to decide the pharmacokinetic parameters on humans for further development [24].

\subsubsection{Phase 1 Clinical Studies}

Classic phase 1 studies of a new drug examine the short-term toxicity for the first time in human subjects and seek to define a maximum tolerated dose. Long-term and cumulative toxicities are not studied and may not be identified at this stage of investigation. A further goal of phase 1 trials is to recommend a dose range for efficacy testing in later, phase 2 trials [25].

The primary aim of a phase I trial investigating cytotoxic agents is to establish the maximum tolerated dose and describe the dose-limiting toxicities. The overall probability of response is up to 6\%. Moreover, the responses (mainly partial responses) are observed within the range from 80 to $120 \%$ of recommended dose in later trials. This low response rate is far from patient expectancy: over $85 \%$ of patients participating in phase I studies expect at least a stabilization of their disease. Most cytotoxic phase I protocols require a minimal life expectancy estimated at 3 months, which is a minimum period of observation for the evaluation of side effects. And this 3-month period is necessary to clearly differentiate the morbidity caused by the advanced disease from that caused by the new cytotoxic agent. But there are no clear or reliable guidelines for life expectancy among this population [26].

\subsubsection{Phase II}

Once the initial safety of the drug has been established, the drug will undergo a phase II trial. The purpose of a phase II trial is to investigate the short-term safety and therapeutic efficacy of the drug in patients with the disease or condition that the drug is intended to treat. Patients are given the different drug dosages found to be safe in the phase I trial, allowing the drug's efficacy and adverse effects to be compared between different dosages. When the efficacy and safety of the drug have been demonstrated in patients with the disease or condition that the drug is intended to treat, the drug will proceed to a phase III trial [27].

\subsubsection{Phase III}

Phase III clinical trials are the gold standard to demonstrate the effects of an experimental therapy compared to standard therapy for a disease of interest. The first step when planning a phase III trial is to specify the statistical hypothesis that the trial purports to test, which is usually that the experimental therapy provides some efficacy benefit over standard therapy, without adding significant harm. In a phase III trial, a pre-specified number of patients from the target population are randomized to receive experimental or standard therapy. The patients are treated and followed up according to a protocol that also defines the endpoints of interest, in particular the primary endpoint which is chosen to reflect a clinical benefit of experimental therapy over standard therapy. The trial data are typically monitored by an independent committee who may recommend stopping the trial early, if appropriate. The benefit of experimental therapy over standard therapy, if any, may be observed across all patients, or may be confined to a subset of patients [28].

\subsubsection{Phase IV}

Pharmacovigilance is the term used in Europe describing the ongoing evaluation of the safety of the drug in the postmarketing period; it is a requirement that all pharmaceutical companies with a post marketed product must comply. The drug company will also provide periodic safety update reports on the new drug after its approval. Post-marketing or safety surveillance trials are sometimes referred to as phase IV clinical trials. Harmful effects discovered during phase IV trials can lead to the withdrawal of the drug from the market as seen in the example of rofecoxib (Vioxx) and cerivastatin (Lipobay, also known as Baycol in the United States) [29].

\subsubsection{Phase V}

A new term used in the literature, is also termed as "translational research" to refer the effectiveness of a communitybased research study. It is used to find the interrogation of a new clinical treatment into a large number of public health practices. Generally, the Phase V trials have been considered as the "field research" and it is particularly designed to test generalization of the mechanism to a large sample [30]. 


\section{Conclusion}

A new drug development is a long way proceeds through various phases over many years. It is including testing in vitro, in vivo, pre-clinical and finally clinical trial phases to get into final approval. All of the phases provide detailed explanation and also give us clear views on efficacy, safety and side effects of drugs on human body.

\section{Compliance with ethical standards}

\section{Acknowledgments}

The research work was supported by the Annual Development Program (ADP) of Bangladesh Government. The title of the project is: "Strengthening of existing gamma source of Bangladesh Atomic Energy Commission". Special thanks to The Ministry of Science and Technology and The Ministry of Planning, Government of the People's Republic of Bangladesh.

\section{Disclosure of conflict of interest}

The authors declares that there is no conflict of interest regarding the publication of this paper.

\section{References}

[1] SK Manirul Haque and Elaref S. Ratemi. Drug Development and analysis review. Journal of Pharmaceutical Chemistry Journal. 2017; 50: 837-850.

[2] Shu-Feng Zhou and Wei-Zhu Zhong. Drug Design and Discovery: Principles and Applications. Journal of Molecules. 2017; 22: 279-285.

[3] Joseph A. DiMasi. Ronald W Hansen, Henry G. Grabowski. The price of innovation: new estimates of drug development costs. Journal of Health Economics. 2003; 22: 151-185.

[4] Sandra Kraljevic, Peter J.Stambrook, Kresimir Pavelic, Accelerating drug discovery. European Molecular Biology Organization (EMBO) reports. 2004; 5: 837-842.

[5] Jannatul Ferdows, Tamanna Tasnim and Shayeda shanjeeda Akter. Drug Discovery and development. International Journal of Current Research. 2018; 10: 73682-73684.

[6] Sahoo Biswa Mohan, Dinda S.C., Ravi Kumar B.V.V., Panda J.R. Computational Approaches for Drug Design and Discovery Process. Journal of Current Parma research. 2012; 2: 600-611.

[7] Michael Dickson and Jean Paul Gagnon. Key Factors in the rising cost of new drug discovery and development. Journal of Nature Reviews Drug Discovery. 2004; 3: 417-429.

[8] Richard C. Mohs, Nigel H. Greig, Drug discovery and development: Role of basic biological research. Journal of Alzheimer's \& Dementia: Translational Research \& Clinical Interventions. 2017; 10: 1-7.

[9] Amol B. Deore, Jayprabha R. Dhumane, Hrushikesh V Wagh, Rushikesh B. Sonawane, The Stages of Drug Discovery and Development Process. Asian Journal of Pharmaceutical Research and Development. 2019; 7: 62-67.

[10] JP Hughes, S Rees, SB Kalindjian, KL Philpot. Principles of early drug discovery. British Journal of Pharmacology. 2011; 162: 1239-1249.

[11] Isabella Gashaw, Peter Ellinghaus, Anette Sommer and Khusru Asadullah. What makes a good drug target? Drug Discovery Today. 2011; 175: S24-S30.

[12] Glenn E Croston. The utility of target-based discovery. Expert Opinion on Drug Discovery. 2017; 12: 1-3.

[13] Henning SW, Beste G. Loss-of-function strategies in drug target validation. Current Drug Discovery Technology. $2002 ; 17-21$.

[14] John GH, Martyn NB, Bristol-Myers S. High throughput screening for lead discovery. Burger_s Medicinal Chemistry and Drug Discovery, 6 th edition, Drug Discovery and Drug Development, Wiley Press. 2002; 2: 37-70.

[15] Patidar AK, Selvam G, Jeyakandan M, Mobiya AK, Bagherwal A, Sanadya G, Mehta R. Lead Discovery and lead optimization: A useful strategy in molecular modification of lead compound in analog design. International journal of drug design and discovery. 2011; 2: 458-463. 
[16] V Umashankar, S Gurunathan. Drug discovery: An appraisal, International journal of pharmacy and pharmaceutical sciences. 2015; 7: 59-66.

[17] Thomas J. DiFeo, Ph.D. Drug Product Development: A technical review of chemistry, manufacturing, and controls information for the support of pharmaceutical compound licensing activities. Journal of Drug Development and Industrial Pharmacy. 2003; 29: 939-958.

[18] Pratibha Muntha. Drug Discovery and Development. Journal of Pharmacy and Pharmaceutical Sciences. 2016; 5: 135-142.

[19] Nalaka Gooneratne. Overview of drug development. Academic Entrepreneurship for Medical and Health Scientists. 2019; 1: 1-9.

[20] Krishnasis Chakraborty, KavitaYadav. Drug approval process in US, Europe and India and its regulatory requirements. International Journal of Drug Regulatory Affairs. 2018; 6: 31-39.

[21] Craig A. Umscheid MD, MSCE, David J. Margolis MD, PhD, MSCE and Craig E. Grossman MD, PhD. Key Concepts of Clinical Trials: A Narrative Review. Journal of Postgraduate Medicine. 2015; 123: 194-204.

[22] Ankur Rohilla, Ravi Kumar Singh, Deepti Sharma, Phases of Clinical Trials. International Journal of Pharmaceutical, Chemical and biological sciences. 2013; 3: 700-703.

[23] SB Thorat, SK Banarjee, DD, Gaikwad SL, Jadhav RM, Thorat. Clinical Trial: A Review. International Journal of Pharmaceutical Sciences Review and Research. 2010; 1: 101-106.

[24] The Lancet, Phase 0 trials: a platform for drug development? Lancet 2009; 374: 176.

[25] Jennie. Muglia and John. Di Giouanna! Phase I clinical trials, Journal of Cutaneous Medicine and Surgery. 1998; 2: 236-241.

[26] Nicolas Penel , Marie Vanseymortier, Marie-Edith Bonneterre , Stéphanie Clisant, Eric Dansin, Yvette Vendel , Régis Beuscart, Jacques Bonneterre. Prognostic factors among cancer patients with good performance status screened for phase I trials. Springer Science, 2007; 26: 53-58.

[27] Philip Sedgwick. What are the four phases of clinical research trials? BMJ, 2014; 348: 1-2.

[28] Marc Buyse, Phase III design: principles, (C) Chinese Clinical Oncology. 2016; 5: 1-13.

[29] Nihad A.M. Tamimi Peter Ellis, Drug Development: From Concept to Marketing. Journal of Nephron Clinical Practice. 2009; 113: 125-131.

[30] Christopher P. Adams and Van V. Brantner. Estimating the cost of new drug development: Is It Really $\$ 802$ million?, Health affairs. 2006; 25: 420-428. 